\title{
32. Luminous Beads of Metal Particles Sputtered by Disruptive Discharge in Magnetic Field.
}

\author{
By Hantaro Nagaoka and Tetsugoro Futagami. \\ Institute of Physical and Chemical Research
}

(Rec. March 9, 1928. Comm. by H. NAGaOKA, M.I.A., March 12, 1928.)

In our experiments on the sputtering of metals by disruptive discharge, using heavy current (of order kiloamp.) passing through the electrodes in point contact, the sputtering is greatly increased when the discharge takes place in strong magnetic field. The effect of the field is to give high speed to the particles which are generally charged. Instances of such sputtering have already been communicated ${ }^{1)}$ to the Academy. On examining the particles, they are mostly spherical, but occasionally ellipsoidal or pear-shaped (Fig.'s 1, 2, 3, 5). This is naturally to be expected, as they are in the molten state, and probably vibrating owing to intense heating and violent motion of the discharge. The form is then given by

$$
r=\sum A_{n} P_{n}(\cos \theta)
$$

where $r$ denotes radius vector and $P_{n}(\cos \theta)$ zonal harmonics. The spherical form corresponds to $n=1$, ellipsoidal to $n=2$, and pear-shaped to $n=3, \ldots$ Most of the particles, as picked up after the discharge, are solid in the interior, but some spheres of brown cerium oxide are only shells like those of eggs (Fig. 4). The extraordinary sputtering (Fig. 8) exhibited by the element seems to be due to sudden exhalation of occluded gases.

The elements which are oxidisable give out particles with the oxides formed on the surface; when there are different oxides, as with tungsten, the surface of a particle is usually covered with only one kind of the oxides, although all forms are found on sputtered particles.

The special feature of the tracks of particles are the beads to be found on photograms of different elements. Sometimes the interval between the beads gradually decreases as with $\mathrm{Mg}$ (Fig. 6) and continue for sometime in that state. This may be due to slackening of speed or

1) Nagaoka a. Futagami: Proc. Imp. Acad. 3 (1927) 499, 643. 
curvature of the path. With Ce and W, they occur at nearly equal intervals, and may be counted by more than a hundred. In such cases the track is nearly straight, and is especially so in W and Mo (Fig. 8 and 9). Ca particles show beads which are sometimes strong and sometimes weak without showing regularity. They are usually present in the forks. The appearance of beads is not limited to sputtered particles, but we have instances in carbon particles issuing from charcoal fire blown by bellows (Fig. 10). In this case the interval increases with time; this may also be the case with sparks of metal particles from a grinder. Another case is found in beaded lightning, though the luminiscence is found, not in particles, but in air.

The question how the beads are formed is not easy to solve, but various suggestions to explain the phenomenon can be given. There is no doubt that light emitting particles are rotating; if only one side be luminous, the beads may be the effect of exposing the lighted and the dark face alternately to the camera during the translation and rotation, but as the particles are very small, the heating cannot be confined to one side of the particle only.

The second suggestion is the attachment of electrons to the hot particles. Owing to the emission of thermions, they are mostly positively charged ; the luminosity is but transient, but on every attachment of electrons they become luminescent. As already mentioned, W shows great number of beads, and as the liberation of thermions is greatest among ordinary metals, the subsequent recovery must be numerous and gives rise to a large number of beads.

The third suggestion is the formation of oxides on the surface of sputtered particles. With oxidisable elements, the passage of fine hot particles through air causes oxidation; thin oxide film covers the surface temporarily; this process recurs so often till the temperature is inadequate to form the coating or to give luminosity. With highly oxidisable substance as $\mathrm{Ca}$, the surface coating of the oxide may fall off, and cause great luminosity in the track; consequently the beads are not regular. The curved path may sometimes cause such effects ; perhaps the beads observed with sputtered $\mathrm{Al}$ may be traced to this cause. Owing to the absence of beads in particles of noble metals, it is difficult to say which of these suggestions is the most plausible, but the last seems to meet the various cases in its different features. Perhaps other reasons can be adduced to explain the curious phenomenon; we have only cited the above, imperfect as the suggestions are, to be one of the causes. 


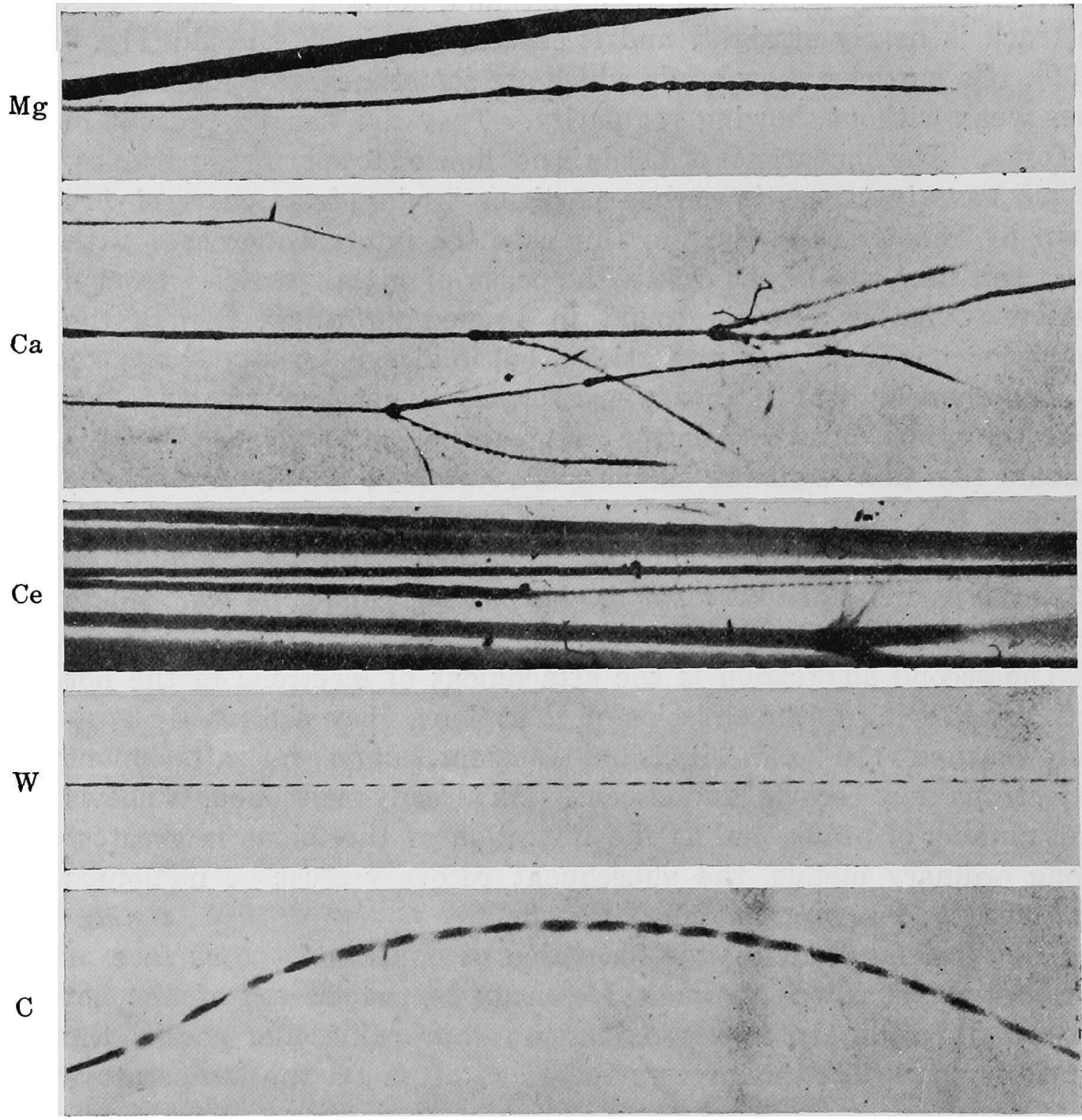

Fig. 6

Fig. 7

Fig. 8

Fig. 9

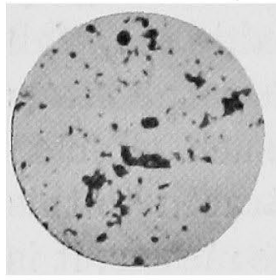

$\mathrm{Mg} \times 10$

Fig. 1

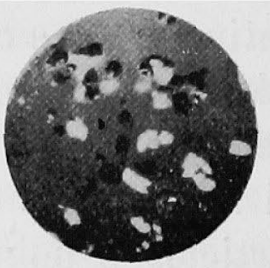

$\mathrm{Ca} \times 10$

Fig. 2

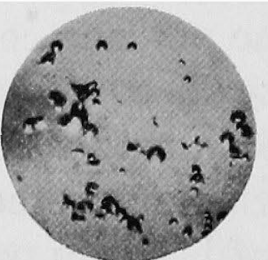

Ce $\times 10$

Fig. 3

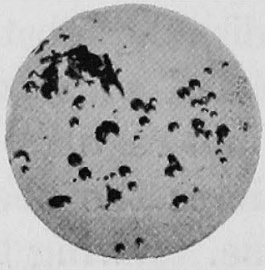

$\mathrm{W} \times 10$

Fig. 5
$\mathrm{Ce} \times 35$

Fig. 4 